\title{
Heteronomous DNA
}

Struther Arnott*, R.Chandrasekaran, I.H.Hall and L.C.Puigjaner

Department of Biological Sciences, Purdue University, West Lafayette, IN 47907, USA

Received 8 February 1983; Revised and Accepted 27 April 1983

ABSTRACT

A fibrous form of poly $d(A)$ : poly $d(T)$ has a heteronomous secondary structure which is the first to be confirmed for a polynucleotide duplex: although both chains are 10, helices, mutually hydrogen-bonded in the standard (Watson-Crick) fashion, each has a quite different conformation. One chain -- probably poly $\mathrm{d}(\mathrm{A})$-- has $C 3^{\prime}$-endo-puckered furanose rings characteristic of the A family of polynucleotide secondary structures while the other -- probably poly $d(T)$-- has the $C 2$ '-endo-puckered rings of the $B$ family. Since analogous heteronomous structures could be assumed by DNA-DNA or DNA-RNA duplexes containing more general base sequences the polymorphic range of polynucleotide double-helices may be even greater than we have come to suppose.

\section{INTRODUCTION}

Until now all successful models of fibrous polynucleotide duplexes containing Watson-Crick base-pairs have been analogous to the paradigmatic version (1) in having identical, anti-parallel chains. This is to say that they have diad axes perpendicular to their axes of screw symmetry. Diad axes lie midway between successive base-pairs in duplexes which have a dinucleotide as the repeated structural motif such as the left-handed allomorphs of $\left(\begin{array}{lll}2 & 2 & 65\end{array}\right)$ poly $d(G C)$ :poly $d(G C)$ and $\left(27_{6}\right)$ poly $d\left(A s^{4} T\right)$ :poly $d\left(A s^{4} T\right)(2)$, and the wrinkled, right-handed allomorphs of $\left(2 \quad 2 \quad 4_{1}\right)$ poly $d(A T)$ :poly $d(A T)$ and $\left(25_{1}\right)$ poly $d(G C)$ :poly $d(G C)(3)$. Duplexes like classical A and $B$ DNA, which have a mononucleotide as the repeated structural motif and more general base sequences, have additional diads in the mean planes of their base-pairs. The symmetry elements in these structures are, of course, only approximate since $A: T, T: A, G: C$ and $C: G$ basepairs are only quasi-isomorphous.

Clearly there is no absolute requirement that polynucleotide chains in multi-stranded complexes be conformationally identical. The triplex structures formed by $\operatorname{poly}(U): \operatorname{poly}(A): \operatorname{poly}(U)(4)$, by 
poly $d(T)$ :poly $d(A)$ :poly $d(T)(5)$, and by poly(I):poly $(A): \operatorname{poly}(I)(6)$, illustrate this clearly and one of the models (23) for poly $r(A)$ :poly $d(T)$ with different furanose conformations in its two chains demonstrates this even more emphatically. Thus in duplexes where the complementary strands are rather different chemically we might expect that the traditional diadic or quasi-diadic symmetry would sometimes be abandoned. We have found this incontrovertibly to be the case in a polycrystalline, fibrous form of poly $d(A)$ :poly $d(T)$. In this instance the information derived from the $X$-ray diffraction pattern (in the form of unit cell dimensions and the intensities of the Bragg reflexions) is sufficient not only to eliminate more conventional models but also to force the conversion of one of these unsatisfactory models into an acceptable model of a non-traditional kind during a least-squares refinement.

POLY D(A):POLY D(T)

Fibers of poly $d(A)$ :poly $d(T)$ were first analysed by Arnott and Selsing (5) who obtained $X$-ray patterns different from the classical $A$ and $B$ patterns of general sequence DNA. One pattern $(\alpha)$ (Fig. 1a), obtained above $85 \%$ relative humidity, indicated 10 -fold helical molecules with a pitch $(P=3.29 \mathrm{~nm})$ somewhat less than that $(3.37 \mathrm{~nm})$ of the common $10_{1} B$ form observed with DNA from calf thymus, salmon sperm, etc. In the $\alpha$ form the poly $d(A)$ :poly $d(T)$ molecules are packed in a screw-disordered, quasitrigonal fashion with long axes $2.28 \mathrm{~nm}$ apart ( $F i g .2 a)$. A second ( $\beta$ ) pattern (Fig. 1b), obtained below 77\% r.h., indicated apparently isomorphous 10-fold helical molecules $(P=3.24 \mathrm{~nm})$ packed in a fully crystalline fashion.

Arnott and Selsing (5) assumed that the $\alpha$ and $\beta$ forms indeed contained the same allomorph and that this was a minor variant of B DNA. They developed a provisional molecular model with $2210_{1}$ symmetry and fitted it to the Bragg diffraction data from the $\alpha$ form. At that time there was no pressing reason to consider alternative models. Since then, however, a more protean view of the structure of DNA has evolved. This prompted us to review the structure of poly $d(A)$ :poly $d(T)$ using the richer data set from the $B$ pattern. When we did this, we discovered that we could devise neither an $A$ nor a $B$ model with the traditional set of diad and screw symmetry elements, whether right-handed or left-handed, which could be accommodated in the unit cell. Only after we removed the constraint that the two chains had to be conformationally identical did we find a model that could be refined to a structure with a sterically unexceptional crystal arrangement and a good fit 

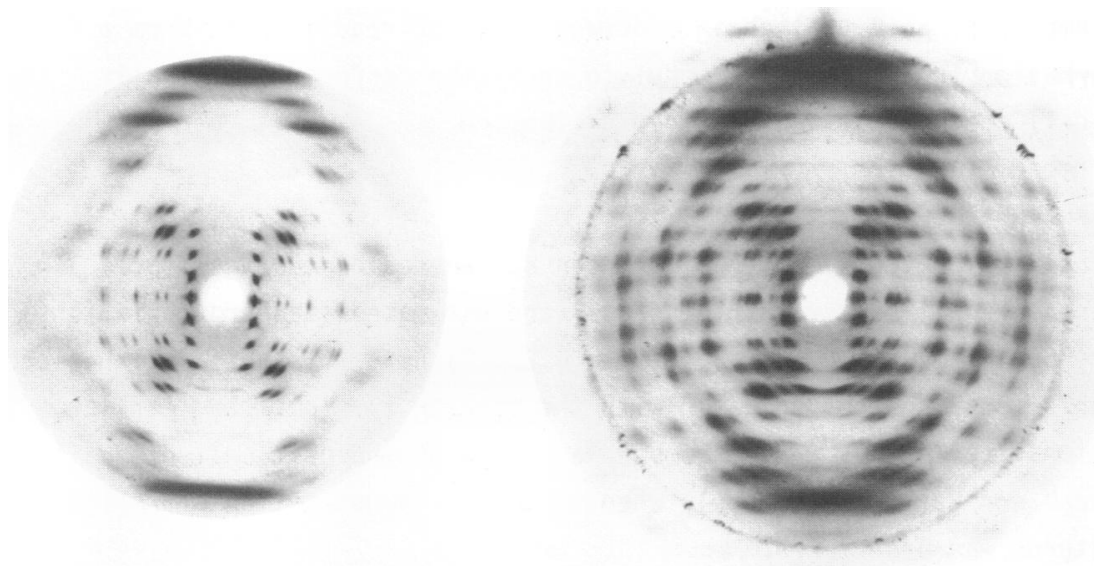

(a)

(b)

Figure 1: X-ray fiber diffraction patterns of poly $d(A)$ :poly $d(T)$ :

(a) the quasi-hexagonal, screw-disordered, $\alpha$ form obtained at $92 \%$ r.h.;

(b) the monoclinic, poly-crystalline, $\beta$ form obtained at $71 \%$ r.h.

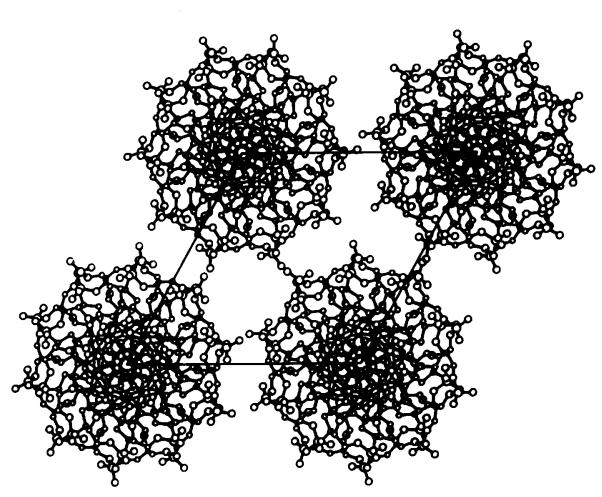

(a)

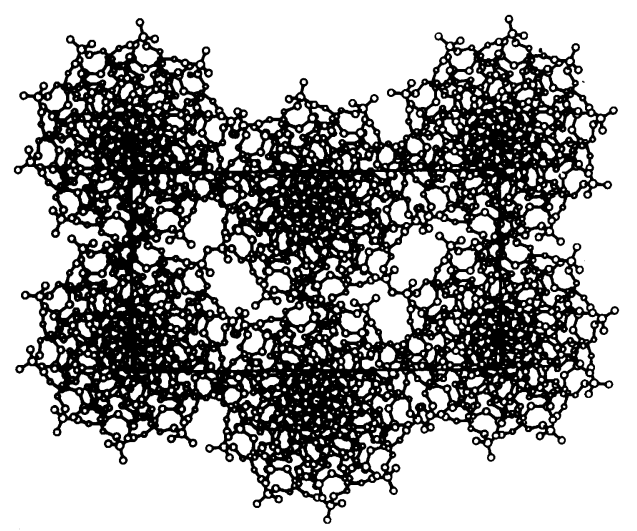

(b)

Figure 2: The molecular packings of heteronomous duplexes of poly $d(A)$ :poly $d(T)$ : (a) in the a form, where the molecules have random orientations due to screw-disordering, four neighbouring molecules form a rhombus of side $2.28 \mathrm{~nm}$ and acute angle $60^{\circ}$; (b) in the more crystalline $\beta$ form, quartets of molecules also have a rhombic arrangement but with side $1.87 \mathrm{~nm}$ and acute angle $72^{\circ}$. 
with the observed intensities of diffraction. Moreover during the process of refinement the poly $d(A)$ chain underwent a major readjustment from a $B-1$ ike conformation in the starting model to an A-like conformation finally. The poly $d(T)$ chain, however, remained $B-1$ ike.

\section{CRYSTAL PACKING}

The X-ray pattern of the $B$ form can be indexed on the basis of a rectangular unit cell with dimensions (and estimated standard deviations) $\underline{a}=1.865(2) \mathrm{nm}, \underline{\mathrm{b}}=3.548(2) \mathrm{nm}, \underline{c}=3.233(3) \mathrm{nm}$. This unit cell has one dimension doubled when compared with the version (5) published earlier. The additional reflexions which prompted a review of the unit cell dimensions are all weak and were detected only when intensive investigation of well-exposed $B$ patterns was undertaken.

Interestingly $\underline{b} / 2 \underline{a}=\cos \pi / 10$ and the relative positions of the two molecules in each unit cell turn out to be $(0,0,0)$ and $(\underline{a} \sin (\pi / 10)$, $\underline{b} / 2(=\underline{a} \cos (\pi / 10)), w)$ implying that quartets of molecules are arranged at the corners of a rhombus of side $1.87 \mathrm{~nm}$ and acute angle $2 \pi / 5$ (Fig. $2 \mathrm{~b}$ ). Analogous arrangements are observed with other DNAs which have 10 or 5 -fold screw axes (3).

\section{REF INEMENT OF MODELS AND ARBITRATION BETWEEN COMPETITORS}

The best molecular and crystal models of each form considered were obtained by the method of Linked Atom Least Squares $(7,8)$. In this LALS method, the main molecular variables were the $2 \times 6$ conformation angles associated with the backbones of each $d A$ and $d T$ nucleotide, and the 2 glycosylic angles describing the orientation of each type of base with respect to its attached deoxyribose. Tilts and propeller twists of basepairs were unrestrained variables. Additional molecular variables were the conformation angles of the furanose rings and their endocyclic bond angles which were allowed to vary in a stiffly elastic fashion. Packing variables were the positional and orientation parameters for each molecule. $X$-ray variables were a scale factor $(K)$ for the observed structure amplitudes and an isotropic attenuation factor (B) for the calculated amplitudes.

The variables were adjusted so as to minimize (in least-squares fashion) the expression

$$
\begin{aligned}
\Omega & =\sum \omega_{{ }_{m}}^{\Delta F_{m}^{2}}+\sum k_{\underline{i}} \Delta d_{i}^{2}+\sum \lambda_{h^{G}} \underline{h} \\
& =\underline{x}+\underline{c}+\underline{L} \text {, say. }
\end{aligned}
$$


$\underline{X}$ involves the differences between the observed $X$-ray amplitudes and those calculated from the model structure. $\underline{c}$ involves both the close, non-bonded interatomic distances, $d_{j}$, which are driven towards some acceptably large value ${ }_{0} d_{j}$, and the conformation and bond angles which are elastically restrained. L involves constraints $G_{h}$ which become zero when residue connectivity and furanose ring closure have been achieved.

Competing molecular and crystal structures can be assessed for significant differences by using $\Omega, \underline{X}$ or $\underline{C}$ as statistics in Hamilton's test $(9,10)$. Details of this and other strategies which are now quite commonplace in the application of LALS to fiber diffraction analyses have been discussed many times before (e.g. 10,11) and will not be described further.

EMERGENCE OF A HETERONOMOUS MODEL

A contemporary version of the Arnott and Selsing B-like, $2210_{1}$ model with $P=3.29 \mathrm{~nm}$ is illustrated in $\mathrm{Fig}$. $3 \mathrm{~d}$. A relaxed $10_{1}$ version in which the poly $d A$ and poly $d T$ chains have similar, but not identical, conformations is shown in Fig. 3c. Unlike the 1974 models, the current ones include all the hydrogen atoms. These are not very important $X$-ray scatterers but contribute significantly to the steric properties of the models. There is no significant difference between these two models insofar as fitting the $X$-ray data from the semi-crystalline $\alpha$ form is concerned. Interestingly the root mean squared difference in the backbone conformation angles of the two chains in the relaxed model is only $\left\langle(\Delta \theta)^{2}\right\rangle^{1 / 2}=3.2^{\circ}$.

The lateral separation of molecular centers is quite large $(2.28 \mathrm{~nm})$ in the $\alpha$ form but only $1.87 \mathrm{~nm}$ in the crystalline $\beta$ form. In this form both the $2210_{1}$ and $10_{1} B$ models lead to extremely short non-bonded interatomic distances between neighbouring molecules. (Distances are considered overshort when they are more than $0.04 \mathrm{~nm}$ less than the sum of the van der Waals radii of the two atoms involved. Hydrogen atoms are important.) This steric compression can be relieved somewhat but the resulting structures all have unacceptably high values $(R>0.4)$ of the crystallographic discrepancy index $R=\Sigma\left|\Delta F_{-m}\right| / \Sigma_{0} F_{m}$. The progress of the least-squares refinements was oscillatory and convergence slow as would be expected for severely flawed models.

Persistent refinement of one $10_{1} B$ model eventually produced a structure (Fig. 3a, Table 1) free of overshort interatomic distances within and between molecules, and possessed of a creditably low value of $R(0.30)$ for the 113 measurable reflexions (Table 2 ). The crucial event leading to 


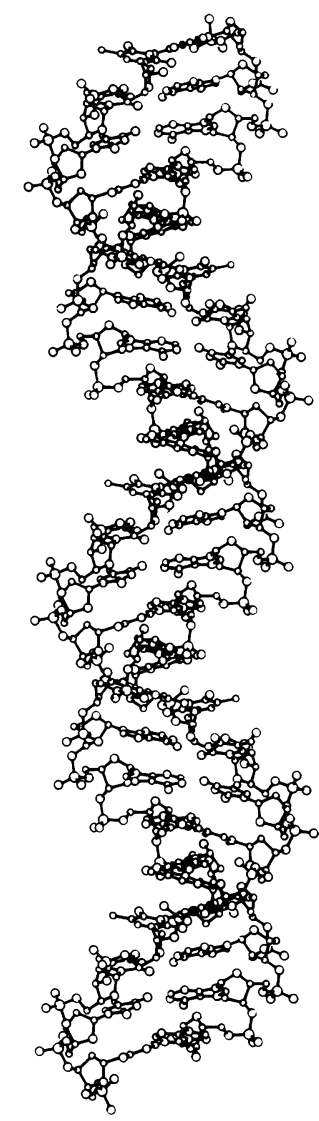

a

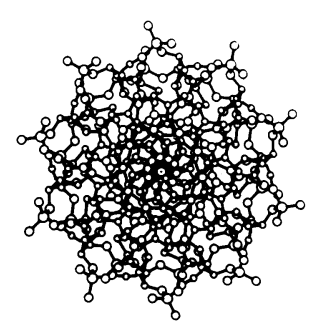

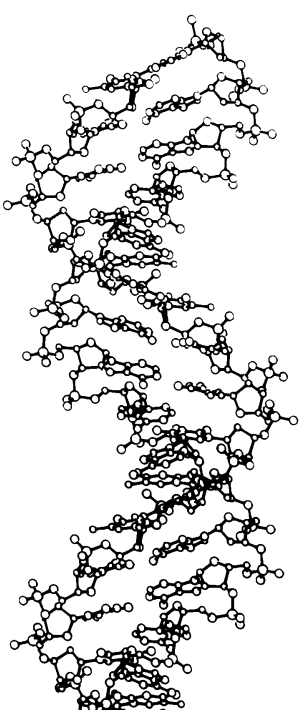

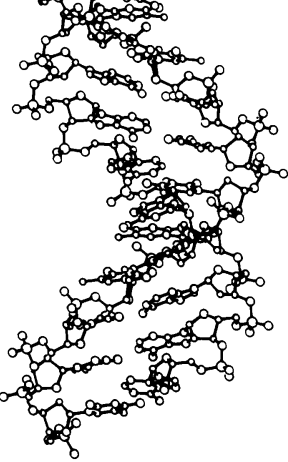

b

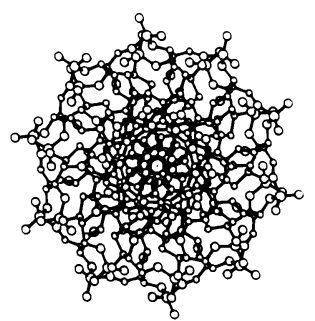

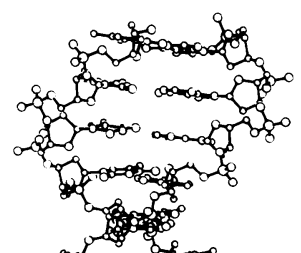

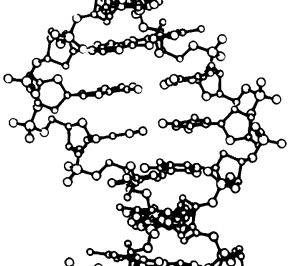

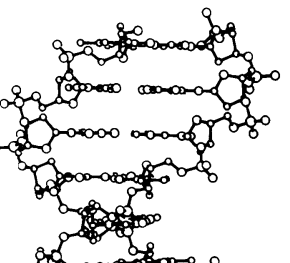<smiles>[Te]C1CCCCC1</smiles>

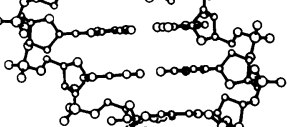

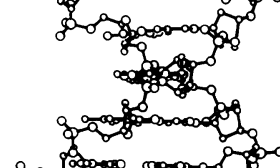
-

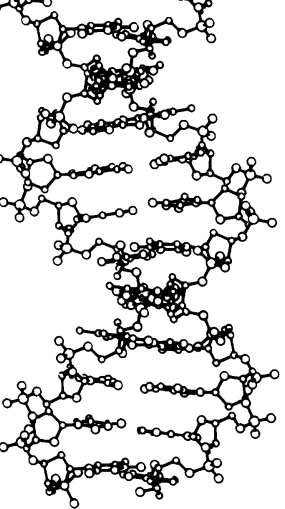

C

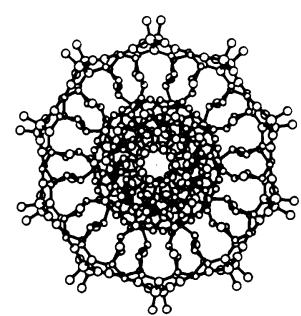

Figure 3: The morphology of the heteronomous and diadically symmetric duplexes of poly $d(A)$ :poly $d(T)$. Views normal to the helix axes are shown at top and the corresponding helix axis projections are underneath. (a) and (b) show similar heteronomous models (each with $A$ and $B$ chains) developed to fit the $\beta$ and $\alpha$ data respectively. (c) shows a mildly heteronomous model with similar but not identical B-like chains. (d) shows a highly symmetrical, $2210_{1}$ model. 
Table 1: Cartesian and cylindrical polar coordinates of the atoms in the molecular asymmetric unit of the $\beta$ form of poly $d(A)$ :poly $d(T)$. Successive nucleotide pairs can be generated by adding multiples of $36^{\circ}$ to $\phi$ and the same multiple of $0.323 \mathrm{~nm}$ to $Z$.

\begin{tabular}{|c|c|c|c|c|c|c|}
\hline GROUP & ATOM & $X(N M)$ & $Y(N M)$ & $Z(N M)$ & $R(N)$ & PHI (DEG) \\
\hline PHOSPHATE & $\begin{array}{l}P \\
01 \\
02 \\
03 \\
05\end{array}$ & $\begin{array}{l}-0.1484 \\
-0.2482 \\
-0.1428 \\
-0.1719 \\
-0.0033\end{array}$ & $\begin{array}{l}0.7754 \\
0.6836 \\
0.9115 \\
0.7853 \\
0.7084\end{array}$ & $\begin{array}{l}0.0675 \\
0.0081 \\
0.0095 \\
0.2254 \\
0.0612\end{array}$ & $\begin{array}{l}0.7895 \\
0.7273 \\
0.9225 \\
0.8039 \\
0.7084\end{array}$ & $\begin{array}{r}100.84 \\
109.95 \\
98.90 \\
102.34 \\
90.27\end{array}$ \\
\hline SUGAR & 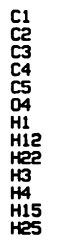 & $\begin{array}{l}0.2533 \\
0.3335 \\
0.2540 \\
0.2299 \\
0.1078 \\
0.2015 \\
0.3203 \\
0.3316 \\
0.4330 \\
0.1576 \\
0.3151 \\
0.1301 \\
0.0817\end{array}$ & $\begin{array}{l}0.4571 \\
0.5143 \\
0.6389 \\
0.6866 \\
0.7764 \\
0.5692 \\
0.4006 \\
0.4425 \\
0.5441 \\
0.6155 \\
0.7407 \\
0.8684 \\
0.8033\end{array}$ & $\begin{array}{r}0.1345 \\
0.0180 \\
-0.0204 \\
0.1229 \\
0.1225 \\
0.2044 \\
0.2009 \\
-0.0652 \\
0.0545 \\
-0.0679 \\
0.1664 \\
0.0664 \\
0.2260\end{array}$ & $\begin{array}{l}0.5226 \\
0.6130 \\
0.6876 \\
0.7241 \\
0.7839 \\
0.6038 \\
0.5129 \\
0.5529 \\
0.6953 \\
0.6353 \\
0.8050 \\
0.8780 \\
0.8075\end{array}$ & $\begin{array}{l}61.01 \\
57.04 \\
68.32 \\
71.49 \\
82.10 \\
70.50 \\
51.35 \\
53.15 \\
51.49 \\
75.64 \\
56.95 \\
81.48 \\
84.19\end{array}$ \\
\hline ADEMINE & 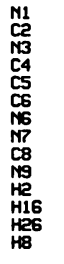 & $\begin{array}{r}0.1078 \\
0.2248 \\
0.2549 \\
0.1466 \\
0.0209 \\
0.0013 \\
-0.1168 \\
-0.0648 \\
0.0106 \\
0.1402 \\
0.3004 \\
-0.1248 \\
-0.1968 \\
-0.0230\end{array}$ & $\begin{array}{r}-0.0211 \\
0.0307 \\
0.1553 \\
0.2349 \\
0.1965 \\
0.0605 \\
0.0089 \\
0.3057 \\
0.4051 \\
0.3699 \\
-0.0344 \\
-0.0889 \\
0.0683 \\
0.4989\end{array}$ & $\begin{array}{r}0.0156 \\
0.0526 \\
0.0817 \\
0.0699 \\
0.0331 \\
0.0045 \\
-0.0327 \\
0.0319 \\
0.0674 \\
0.0921 \\
0.0592 \\
-0.0519 \\
-0.0413 \\
0.0759\end{array}$ & $\begin{array}{l}0.1098 \\
0.2268 \\
0.2985 \\
0.2769 \\
0.1976 \\
0.0605 \\
0.1171 \\
0.3125 \\
0.4052 \\
0.3956 \\
0.3023 \\
0.1533 \\
0.2083 \\
0.4994\end{array}$ & $\begin{array}{r}-11.09 \\
7.77 \\
31.35 \\
58.03 \\
83.94 \\
88.79 \\
-184.37 \\
101.96 \\
88.49 \\
69.24 \\
-6.54 \\
-144.54 \\
-199.14 \\
92.64\end{array}$ \\
\hline PHOSPHATE & $\begin{array}{l}P \\
01 \\
02 \\
03 \\
05\end{array}$ & $\begin{array}{r}0.0595 \\
0.1263 \\
-0.0571 \\
0.0168 \\
0.1655\end{array}$ & $\begin{array}{l}-0.9591 \\
-1.0994 \\
-0.9702 \\
-0.9066 \\
-0.8618\end{array}$ & $\begin{array}{l}-0.3494 \\
-0.3710 \\
-0.2582 \\
-0.4903 \\
-0.2982\end{array}$ & $\begin{array}{l}0.9709 \\
1.1066 \\
0.9719 \\
0.9067 \\
0.8777\end{array}$ & $\begin{array}{l}-86.49 \\
-83.45 \\
-93.37 \\
-88.94 \\
-79.06\end{array}$ \\
\hline SUCAR & $\begin{array}{l}C 1 \\
C 2 \\
C 3 \\
C 4 \\
C 5 \\
04 \\
\text { H1 } \\
\text { H12 } \\
\text { He2 } \\
\text { H3 } \\
\text { H4 } \\
\text { H15 } \\
\text { HES }\end{array}$ & $\begin{array}{l}0.3095 \\
0.3204 \\
0.4163 \\
0.3614 \\
0.2605 \\
0.2996 \\
0.3995 \\
0.3679 \\
0.2223 \\
0.4083 \\
0.4449 \\
0.2067 \\
0.3130\end{array}$ & $\begin{array}{l}-0.5780 \\
-0.7193 \\
-0.7763 \\
-0.7196 \\
-0.8066 \\
-0.5935 \\
-0.5208 \\
-0.7158 \\
-0.7687 \\
-0.8860 \\
-0.6962 \\
-0.7461 \\
-0.8884\end{array}$ & $\begin{array}{r}-0.1407 \\
-0.0845 \\
-0.1889 \\
-0.3199 \\
-0.3923 \\
-0.2814 \\
-0.1138 \\
0.0147 \\
-0.0902 \\
-0.1902 \\
-0.3876 \\
-0.4668 \\
-0.4440\end{array}$ & $\begin{array}{l}0.6556 \\
0.7874 \\
0.8808 \\
0.8053 \\
0.8476 \\
0.6648 \\
0.6564 \\
0.8048 \\
0.8002 \\
0.9755 \\
0.8262 \\
0.7742 \\
0.9419\end{array}$ & $\begin{array}{l}-61.83 \\
-65.99 \\
-61.80 \\
-63.33 \\
-72.10 \\
-63.22 \\
-52.50 \\
-62.80 \\
-73.87 \\
-65.26 \\
-57.42 \\
-74.52 \\
-70.59\end{array}$ \\
\hline THMAIRE & 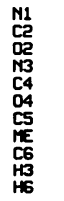 & $\begin{array}{r}0.1884 \\
0.2002 \\
0.3039 \\
0.0859 \\
-0.0361 \\
-0.1315 \\
-0.0387 \\
-0.1678 \\
0.0710 \\
0.0923 \\
0.0669\end{array}$ & $\begin{array}{l}-0.5044 \\
-0.3685 \\
-0.3080 \\
-0.3038 \\
-0.3623 \\
-0.2938 \\
-0.5052 \\
-0.5768 \\
-0.5707 \\
-0.2048 \\
-0.6697\end{array}$ & $\begin{array}{l}-0.0947 \\
-0.0783 \\
-0.0995 \\
-0.0356 \\
-0.0087 \\
0.0290 \\
-0.0289 \\
-0.0019 \\
-0.0703 \\
-0.0229 \\
-0.0836\end{array}$ & $\begin{array}{l}0.5384 \\
0.4194 \\
0.4326 \\
0.3157 \\
0.3640 \\
0.3219 \\
0.5067 \\
0.6007 \\
0.5751 \\
0.2246 \\
0.6730\end{array}$ & $\begin{array}{r}-69.52 \\
-61.49 \\
-45.39 \\
-74.20 \\
-95.70 \\
-114.11 \\
-94.38 \\
-106.22 \\
-82.91 \\
-65.74 \\
-84.30\end{array}$ \\
\hline
\end{tabular}


Table 2: Structure amplitudes for the $B$ form of poly $d(A)$ :poly $d(T)$ : $(A)$ observed and (B) calculated values. M denotes a meridional reflexion and $\mathrm{N}$ a systematic absence. Amplitudes in parentheses indicate reflexions too weak to be measured. In $(A)$ the estimated threshold value is given. In (B) the calculated values are shown. Such reflexions were included in the refinement only when the calculated amplitudes had values greater than threshold. The spot marked * in (A) could not be densitometered due to severe overlap and high background and hence was not included in the refinement. The value of the attenuation parameter for the calculated amplitudes is $B=-0.14 \mathrm{~nm}^{2}$.
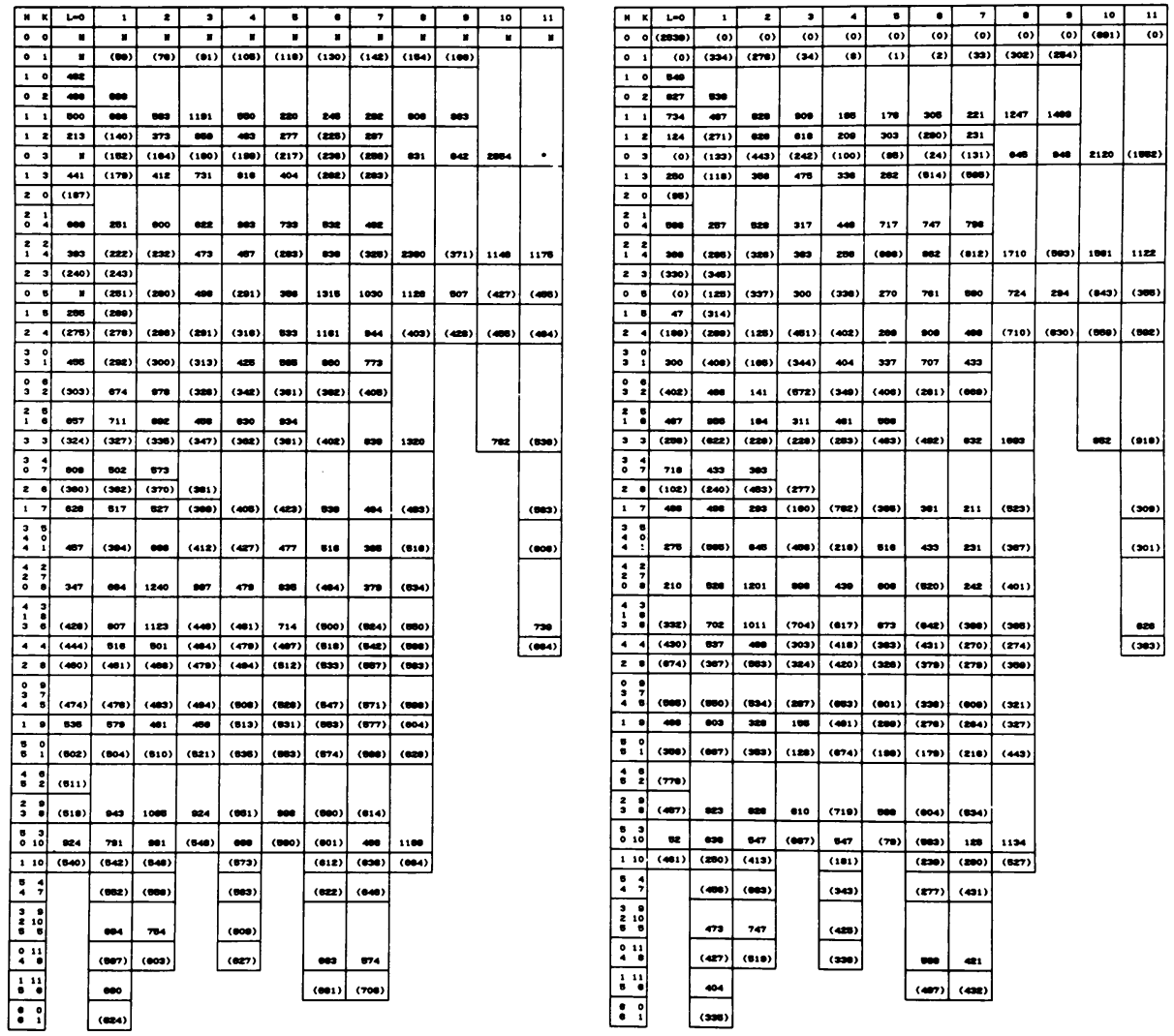

this felicitious outcome was the change in the puckering of the sugar rings of the poly $d(A)$ chain from C2' -endo to $C 3^{\prime}$-endo (Table 3 ).

\section{TESTS OF OTHER MODELS}

The most compelling argument for the credibility of the novel structure for poly $d(A)$ :poly $d(T)$ is that it evolved spontaneously from a quite 
Table 3: The values (and estimated standard deviations) of the molecular parameters for $B$ poly $d(A)$ :poly $d(T)$. Those of $A$ and $B$ DNA are given for comparison with $\mathrm{dA}$ and $\mathrm{dT}$ strands respectively.

\begin{tabular}{|c|c|c|c|c|}
\hline Parameters & A-DNA & ApA & TpT & B DNA \\
\hline $\begin{array}{l}\text { Backbone } \\
\text { Conformations }\left({ }^{\circ}\right) \\
\theta\left(C 4^{\prime}-C 3^{\prime}-03^{\prime}-P\right) \\
\theta\left(C 3^{\prime}-03^{\prime}-P-05^{\prime}\right) \\
\theta\left(03^{\prime}-P-05^{\prime}-C 5^{\prime}\right) \\
\theta\left(P-05^{\prime}-C 5^{\prime}-C 4^{\prime}\right) \\
\theta\left(05^{\prime}-C 5^{\prime}-C 4^{\prime}-C 3^{\prime}\right) \\
\theta\left(C 5^{\prime}-C 4^{\prime}-C 3^{\prime}-03^{\prime}\right) \\
\text { Glycosyl ic } \\
\text { Conformations }\left(^{\circ}\right) \\
\theta\left(C 2^{\prime}-C 1^{\prime}-N 9^{\prime}-C 4\right) \\
\theta\left(C 2^{\prime}-C 1^{\prime}-N 1^{\prime}-C 2\right) \\
\text { Furanose } \\
\text { Conformations }\left(^{\circ}\right) \\
\theta\left(C 4^{\prime}-04^{\prime}-C 1^{\prime}-C 2^{\prime}\right) \\
\theta\left(04^{\prime}-C 1^{\prime}-C 2^{\prime}-C 3^{\prime}\right) \\
\theta\left(C 1^{\prime}-C 2^{\prime}-C 3^{\prime}-C 4^{\prime}\right) \\
\theta\left(C 2^{\prime}-C 3^{\prime}-C 4^{\prime}-04^{\prime}\right) \\
\theta\left(C 3^{\prime}-C 4^{\prime}-04^{\prime}-C 1^{\prime}\right) \\
\text { Furanose Endocyclic } \\
\text { Bond Angles }\left({ }^{\circ}\right) \\
\tau\left(C 4^{\prime}-04^{\prime}-C 1^{\prime}\right) \\
\tau\left(04^{\prime}-C 1^{\prime}-C 2^{\prime}\right) \\
\tau\left(C 1^{\prime}-C 2^{\prime}-C 3^{\prime}\right) \\
\tau\left(C 2^{\prime}-C 3^{\prime}-C 4^{\prime}\right) \\
\tau\left(C 3^{\prime}-C 4^{\prime}-04^{\prime}\right)\end{array}$ & $\begin{array}{r}-145 \\
-78 \\
-50 \\
172 \\
42 \\
79\end{array}$ & $\begin{array}{r}9(1) \\
-35(2) \\
43(1) \\
-40(1) \\
21(1) \\
\\
\\
107(2) \\
106(2) \\
103(2) \\
96(2) \\
108(2)\end{array}$ & $\begin{array}{r}171(4) \\
-121(5) \\
-41(5) \\
-174(5) \\
43(4) \\
152(3) \\
- \\
147(3)\end{array}$ & $\begin{array}{r}-141 \\
-157 \\
-33 \\
138 \\
33 \\
142\end{array}$ \\
\hline $\begin{array}{l}\text { Dihedral Angle between } \\
\text { Planes in a Base-pair }\left({ }^{\circ}\right) \\
\Delta \gamma\end{array}$ & -6 & & & -13 \\
\hline
\end{tabular}

different structure during the course of an unbiased least-squares refinement designed only to abolish steric compression and at the same time to optimize the fit between the calculated and observed structure amplitudes. In addition, however, the final value of $R$ from the heteronomous model is comparable with that obtained with other highly-hydrated, polycrystalline polynucleotide structures. By itself, of course, this index does not assure us that no other viable structural solutions are possible. Therefore we 
generated and tested a variety of alternative models. Fortunately, the number of $X$-ray data is relatively large. (The field contains 113 measurable reflexions and 150 below threshold reflexions.) Also the unit cell is relatively compact. These two circumstances separately or jointly allow strong discrimination between different possibilities.

Firstly we simply exchanged the $A$ and $T$ bases. This had no effect on the $X$-ray index $R$ since low resolution $X$-ray data cannot discriminate between $A: T$ and $T: A$ base-pairs. There were, however, short interstrand contacts between neighbouring, unpaired $A$ and $T$ bases which persisted even after refinement. For this reason we feel the structure is accurately presented with poly $d(A)$ containing $\mathrm{C} 3^{\prime}$-endo furanose rings and poly $\mathrm{d}(\mathrm{T}) \mathrm{C} \mathrm{C}^{\prime}$-endo.

The two heteronomous molecules in each unit cell are related by a $2_{1}$ axis parallel to $\underline{b}$. An alternative model with a 2 axis parallel to $\underline{a}$ has a high $R$ index $(0.40)$ and very short intermolecular contacts.

Left-handed $\left(10_{9}\right)$ heteronomous models are slim and do not give rise to unacceptable steric compression but in no packing arrangement was $R<0.44$.

Molecular models with parallel or antiparallel chains hydrogen-bonded in the Hoogsteen (12) fashion which is a favorable alternative for $A: T$ are also slim but in no instance produced $R \leqslant 0.42$.

Finally, as we mentioned before, mildly heteronomous models with two similar but unequal A-type chains or two similar but unequal B-type chains have 1 arge radi $i$, unacceptable steric compression and large $R$ indices $(\geqslant 0.42)$.

Independent support for specific features of our exotic model for poly $d(A)$ : poly $d(T)$ comes from Raman spectroscopy: Thomas and Peticolas (24) have shown that from samples of poly $d(A)$ :poly $d(T)$ both $C 2^{\prime}$-endo and C $3^{\prime}$-endo marker bands are produced simultaneously. At higher temperatures, but below the melting-point of the duplex, the $c 3^{\prime}$-endo band disappears.

\section{DISCUSSION OF THE STRUCTURE}

Conformations. Table 3 contains the values of the conformational variables in heteronomous poly $d(A)$ :poly $d(T)$ and in $A$ and $B$ DNA. It is clear that the unlike chains in the new structure are each rather conventional members of their respective conformational genera.

Morphology. That the heteronomous model indeed has a distribution of atoms quite different from a diad-containing competitor is emphasised by the views ( $F$ ig. $3 a, d$ ) down the long axes of these alternative structures.

Viewed perpendicular to their long axes, however, the heteronomous $10_{1}$ 


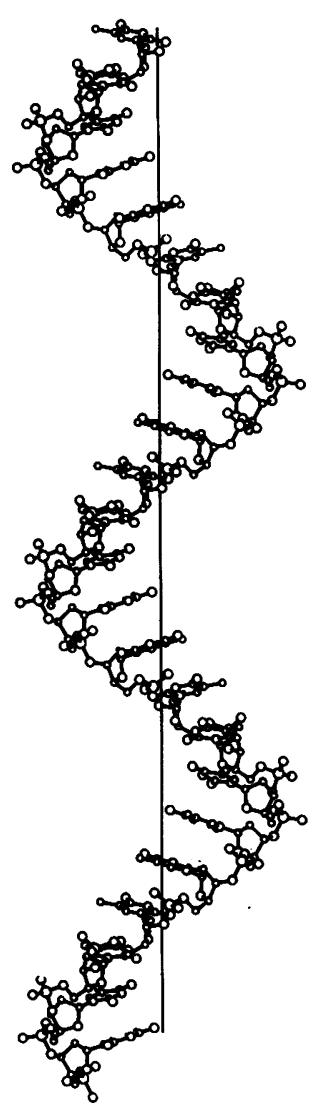

a

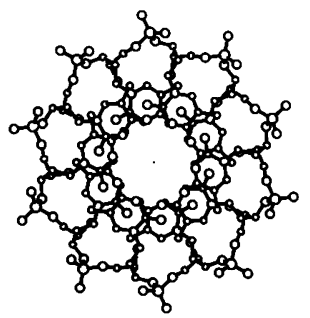

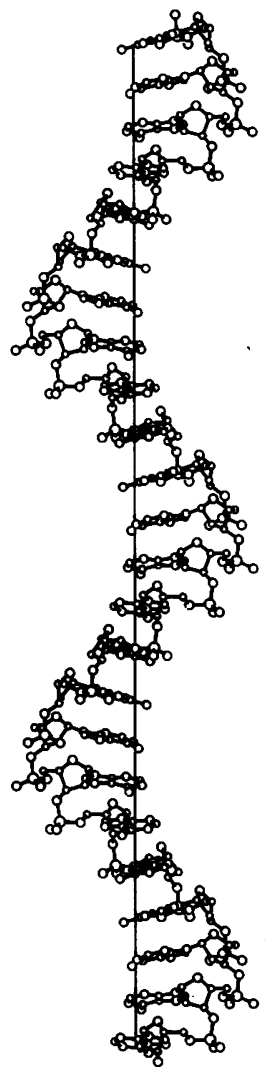

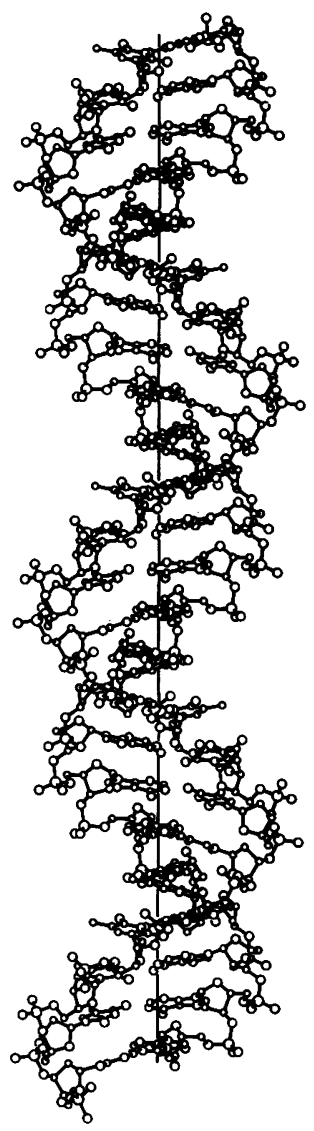

$\boldsymbol{b}$

C

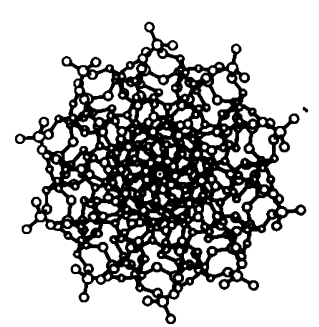

Figure 4: Views normal to (above) and down (below) the vertical helix axes of (a) the $B-1$ ike poly $d(T)$ strand which mates with (b) the A-like poly $d(A)$ strand to produce $(c)$ the heteronomous duplex. 

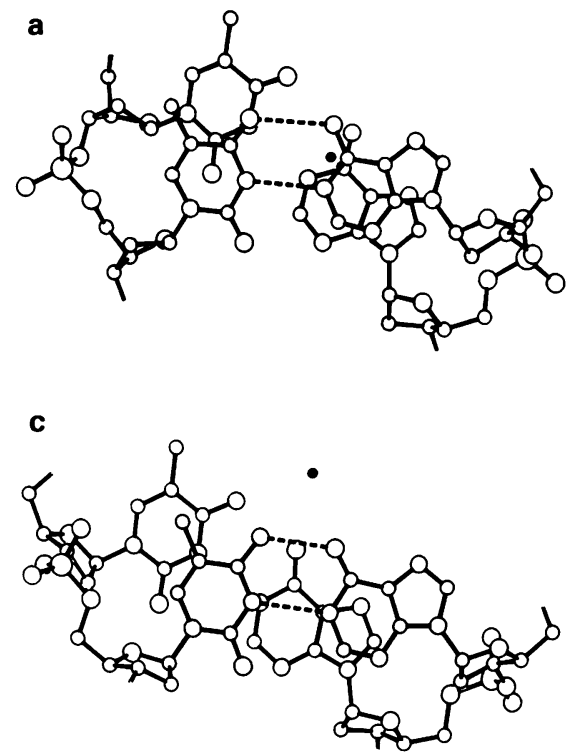

b

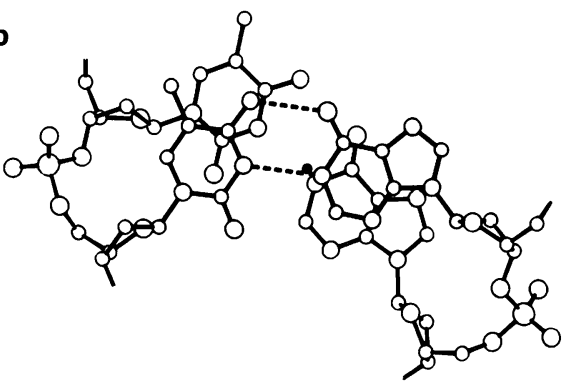

Figure 5: Helix axis projections of dinucleoside phosphate fragments of (a) the heteronomous poly $d(A)$ : poly $d(T)$ duplex, (b) B DNA, and $(c)$ an A DNA allomorph. The base stacking in the heteronomous model is quite similar to that in B DNA although the shapes of the TpT and ApA nucleotides resemble their conformational relatives in $B$ and $A$ DNA respectively.

duplexes (Fig. 3a,b) are not unlike the more symmetrical allomorphs. Thus the conceptual problem associated with a complementary duplex composed of one B-like chain (Fig. 4a) and one A-like chain (Fig. 4b) is difficult only for those who persist in thinking of reconciling chains from the classical $A$ and $B$ DNA structures which have quite different symmetries, pitches and base-tilts.

In Fig. 5 there are close-up views of dinucleoside phosphate fragments from the heteronomous poly $d(A)$ :poly $d(T)$ duplex and corresponding sequences in $B$ DNA and an A-like allomorph (5) with a similar axial translation per nucleotide $(h=0.33 \mathrm{~nm})$. These show that the shape of $d(A p A)$ is pretty much the same in poly $d(A):$ poly $d(T)$ as it is in $A$ DNA. The same is true of $d(T p T)$ in poly $d(A)$ :poly $d(T)$ and in $B$ DNA. What are rather different, of course, are the positions and arrangements of these residues with respect to the helix axes. The differences of radii are apparent in Fig. 5 . Not apparent are the differences in orientation. In heteronomous duplexes the normals to the base planes do not need to make equal angles $(\gamma)$ with the common helix axis and in poly $d(A)$ : poly $d(T) \gamma_{A}=16.5^{\circ}$ is somewhat different from $\gamma_{T}=18.0^{\circ}$. In addition the dihedral angle between the planes of paired bases is quite high $\left(29^{\circ}\right)$ in keeping with the trend observed for A:T pairs in oligomers (13) and polymers (3): in oligomers the base- 
reaches $27^{\circ}$; in poly $d(A T)$ :poly $d(A T)$ it is $21^{\circ}$ compared with $14^{\circ}$ in poly $d(G C)$ : poly $d(G C)$.

Base Stacks. The projections of the various stacked bases in Fig. 5 represent views down the helix axes. It is quite evident that the basestacking in both chains of poly $d(A)$ :poly $d(T)$ resembles that of the same sequences in $B$ DNA but is quite different from what occurs in A-like DNAs. $\alpha$ and $B$ poly $d(A)$ :poly $d(T)$. Our conclusion that $B$ poly $d(A)$ : poly $d(T)$ contains heteronomous duplexes may apply also to the $\alpha$ form. We have tested a variety of molecular models in the $\alpha$ system which is (as we implied earlier) less discriminating than $\beta$ on two counts: its spacious unit cell can accommodate different kinds of duplex more or less equally well; it generates fewer Bragg $X$-ray reflexions. A B-like, $2 \quad 2 \quad 10_{1}$ model (Fig. 3d), a relaxed version of this with similar but not identical $10_{1}$ chains (Fig. $3 C$ ), and an emphatically heteronomous model with one B-like and one $A-l i k e$ chain (Fig. $3 \mathrm{~b}$ ) provide, after refinement, $X$-ray discrepancy indexes $R=0.43,0.43,0.33$ respectively. The first value of $R$ is larger than Arnott and Selsing obtained in 1974 because current modelling technology includes $\mathrm{H}$ atoms on the polynucleotide chains and insists on models with less steric compression. It is true also that steric compression within the heteronomous model is markedly less than is found even in the up-dated B-like models $(\langle\underline{C}\rangle=1.4$ in the former but $\langle\underline{C}\rangle=2.7$ in the latter). Although $X$-ray and steric consideration both favour heteronomous $10_{1}$ over $2210_{1}$ poly $d(A)$ : poly $d(T)$ molecular models in the more hydrated $\alpha$ system, as well as in the $\beta$ system, the number of data in the former are not sufficient to make the conclusion statistically significant.

\section{IMPLICATIONS}

Certainly the persistently anomalous behaviour of poly $d(A)$ :poly $d(T)$ in solution is compatible with its having usually an unconventional structure.

For example, it is possible to predict the ultraviolet circular dichroism spectrum of a DNA of complex sequence by making an appropriate linear combination of CD spectra of DNAs of repeated simple sequences that are fragments of the complex sequence (14). A prerequisite for success is that all the DNAs involved are isomorphous. Interestingly, the measured CD spectrum of poly $d(A)$ : poly $d(T)(15)$ has been shown to be quite anomalous in this connection (16). The predicted spectrum which would form a selfconsistent set with those of other DNAs is dramatically different from the measured version (16). 
Another quite different experiment, involving incomplete scission of poly $d(A)$ :poly $d(T)$ molecules laid down on mica or calcite surfaces, indicates that the pitch of this polymer is significantly different from that of typical DNAs (17).

Further, it has been shown (18) that nucleosomes will not form over a sufficiently (e.g. 80 nucleotide) long segment of poly $d(A)$ :poly $d(T)$ in a recombinant DNA molecule. This implies not only that the segment has a structure substantially different from general sequence DNA but al so that the special structure is sufficiently robust to resist the homogenising effect of flanking sequences. Interestingly, however, smaller (e.g. 20 nucleotide) segments can be accommodated in nucleosomes (18) although poly $d(A)$ :poly $d(T)$ tracts even of this size appear to retain their idiosyncratic secondary structure $(19,20)$. This may imply that such tracts contrive to lie between the sites of closest histone-DNA interaction $(17,21)$ and could therefore play a role in phasing nucleosomes on DNA. Obviously any unconventional DNA secondary structure could produce the phenomena just discussed but it would be surprising if they did not result from heteronomous duplexes resembling (if not identical to) the ones we have been describing.

Monotonous $d(A)_{n}: d(T)_{n}$ tracts need not, of course, be the only sequences with these properties: clearly it is not unthinkable that any oligo $d(P u): 0 l i g o d(P y)$ segment, or any fragment that had approximately such a sequence, might serve equally well.

As mentioned above, Raman spectroscopy (24) has indicated that the precise manner in which poly $d(A)$ :poly $d(T)$ may differ from orthodox DNAs may be its simultaneous accommodation of nucleotides with $C 2$ '-endo and with C3'-endo furanose rings.

Heteronomous duplexes are not confined to polynucleotides with only $A: T$ base pairs. Patterns similar to those of poly $d(A)$ :poly $d(T)$ have been observed also with poly $d(I)$ :poly $d(C)$ and with poly $d(A I)$ :poly $d(C T)(25)$.

DNA-RNA hybrid duplexes are also prime candidates to form heteronomous structures. Although many such hybrids are known to be essentially isomorphous with well-characterized RNA-RNA duplexes which do have diad axes, some have been thought to have heteronomous structures: poly $d(I)$ :poly (C) which has a 10 -fold helix of pitch $P=3.1 \mathrm{~nm}(22)$; poly $(A)$ :poly $d(T)$ which has a pitch $P=3.4 \mathrm{~nm}$ and is thought by some (23) to be a 10-fold helix but by us to be an 11-fold helix isomorphous with poly $d(A)$ :poly (U) (4) in which case the structure is unlikely to be heteronomous. There is a need, 
therefore, for more intensive investigations of the polymorphic range of DNA-RNA hybrids to determine how commonly heteronomous structures occur.

\section{ACKNOWLEDGEMENTS}

We thank Carol Jacobson for typing the text, Bill Boyle for photography and the NIH for a grant (GM17371).

*To whom correspondence should be sent

\section{REFERENCES}

1. Watson, J.D. and Crick, F.H.C. (1953) Nature 171,737-738.

2. Arnott, S., Chandrasekaran, R., Birdsall, D. L., Leslie, A.G.W. and Ratliff, R. L. (1980) Nature 283,743-745.

3. Arnott, S., Chandrasekaran, R., Puigjaner, L. C., Walker, J. K., Hall, I. H., Birdsall, D. L. and Ratliff, R. L. (1983) Nucl. Acids. Res. $11,1457-1474$.

4. Arnott, S. and Bond, P. J. (1973) Nature New Biology 244,99-101.

5. Arnott, S. and Selsing, E. (1974) J. Mol. Biol. 88,509-521.

6. Arnott, S. and Bond, P. J. (1973) Science 181,68-69.

7. Arnott, S. and Wonacott, A. J. (1966) Polymer 7,157-166.

8. Smith, P.J.C. and Arnott, S. (1978) Acta Cryst. A34,3-11.

9. Hamilton, W. C. (1965) Acta Cryst. 18,502-510.

10. Arnott, S. (1980) in Fiber Diffraction Methods, French, A. D. and Gardner, K. H. Eds., Vol 141, pp. 1-30. American Chemical Society, Washington, D.C.

11. Cael, J. J., Winter, W. T. and Arnott, S. (1978) J. Mol. Biol. 125,21-42.

12. Hoogsteen, K. (1963) Acta Cryst. 16,907-916.

13. Fratini, A. V., Kopka, M. L., Drew, H. R. and Dickerson, R. E. (1982) J. Biol. Chem. 257,14686-14707.

14. Gray, D. M. and Tinoco, I., Jr. (1970) Biopolymers 9,223-244.

15. Wells, R. D., Larson, J. E., Grant, R. C., Shortle, B. E. and Cantor, C. R. (1970) J. Mol. Biol. 54,465-497.

16. Arnott, S. (1975) Nucl. Acids Res. 2,1493-1502.

17. Klug, A., Rhodes, D., Smi th, J., Finch, J. T. and Thomas, J. 0. (1980) Nature 287,509-516.

18. Kunkel, G. R. and Martinson, H. G. (1981) Nucl. Acids Res. 9,68696888.

19. Strauss, F., Gaillard, C. and Prunell, A. (1981) Eur. J. Biochem. 118,215222.

20. Peck, L. J. and Wang, J. C. (1981) Nature 292,375-378.

21. Finch, J. T., Brown, R. S., Rhodes, D., Richmond, T., Rushton, B., Lutter, L. C. and Kiug, A. (1981) J. Mol. Biol. 145,757-769.

22. Arnott, S., Chandrasekaran, R., Hall, I. H., Puigjaner, L. C., Walker, J. K. and Wang, Manlin. (1982) in Symposia on Quantitative Biology:

Structures of DNA (Proceedings of the Forty-Seventh Cold Spring Harbor Symposium on Quantitative Biology: Structures of DNA, Cold Spring Harbor Laboratory Publications), Vol XLVII, pp. 53-65.

23. Zimmerman, S. B. and Pheiffer, B. H. (1981) Proc. Natl. Acad. Sci. U.S.A. 78,78-82.

24. Thomas, G. A. and Peticolas, W. L. (1983) J. Am. Chem. Soc. 105,993-996.

25. Leslie, A.G.W., Arnott, S., Chandrasekaran, R. and Ratliff, R. L. (1980) J. Mol. Biol. 143,49-72. 